\title{
Akut suizidal-affektive Störung: Ein systematisches Review
}

\author{
Carola Claus Tobias Teismann \\ Forschungs- und Behandlungszentrum für psychische Gesundheit, Ruhr-Universität Bochum, \\ Bochum, Deutschland
}

\section{Schlüsselwörter}

Suizid · Akut suizidal-affektive Störung · Warnzeichen

\section{Zusammenfassung}

Hintergrund: Die Arbeitsgruppe um Thomas Joiner postuliert ein als acute suicidal affective disturbance (ASAD) bezeichnetes Syndrom, welches durch vier Symptomgruppen (Suizidabsicht, Entfremdung, Hoffnungslosigkeit, Übererregung) definiert sein soll. Ziel des vorliegenden Artikels ist, die Literatur zum ASAD-Syndrom zusammenfassend darzustellen und eine Einschätzung vorzunehmen, inwieweit tatsächlich von einem einheitlichen Syndrom ausgegangen werden kann. Methoden: Im Rahmen einer Literaturrecherche konnten neun Artikel identifiziert werden, die im Zeitraum von 2016 bis 2020 zum Thema publiziert wurden. Ergebnisse und Schlussfolgerungen: Die Befundlage unterstützt den einheitlichen Störungscharakter und die Abgrenzbarkeit der akut suizidal-affektiven Störung von anderen Störungsbildern. Die Aussagekraft der Befundlage ist dadurch eingeschränkt, dass bislang ausschließlich Querschnittsuntersuchungen durchgeführt wurden und keinerlei Befunde dazu vorliegen, ob ASAD tatsächlich suizidalem Verhalten vorausgeht.

2020 S. Karger AG, Base

\section{Acute Suicidal Affective Disturbance: A Systematic Review}

\section{Keywords}

Suicide $\cdot$ Acute suicidal affective disturbance $\cdot$ Warning signs

\begin{abstract}
Background: Joiner and colleagues postulate a syndrome called "acute suicidal affective disturbance" (ASAD), which is defined by four symptom groups (suicide intent, alienation, hopelessness, overarousal). The aim of this article is to summarize the literature on ASAD syndrome and to make an assessment of the extent to which a uniform syndrome can actually be assumed. Methods: A literature search identified nine articles that were published on the issue from 2016 to 2020. Results and Conclusions: The findings support the unidimensional structure of the disorder and the ability to distinguish ASAD from other disorders. The significance of these findings is limited by the fact that so far only cross-sectional study designs have been carried out and there are no findings as to whether ASAD actually precedes suicidal behavior.
\end{abstract}

(c) 2020 S. Karger AG, Basel

\section{Einleitung}

Im Jahr 2018 starben in der Bundesrepublik Deutschland 9'396 Menschen durch einen Suizid [Statistisches Bundesamt, 2020]. Weltweit suizidieren sich nach Angaben der WHO jährlich fast eine Million Menschen [WHO, 2014]. Die Rate von Suizidversuchen wird als bis zu 20fach höher eingeschätzt als die der vollzogenen Suizide [Nock et al., 2012]. Insbesondere in klinischen Populationen finden sich deutlich erhöhte Raten suizidalen Erlebens und Verhaltens [Bernal et al., 2007]. Trotz intensiver Forschungsbemühungen ist es bislang jedoch nicht möglich, Suizidversuche und Suizide - auf der Basis unterschiedlichster Risikofaktoren - wesentlich besser als auf Zufallsniveau vorherzusagen [Franklin et al., 2017]. In 
diesem Zusammenhang kritisieren verschiedene Autoren [Galynker, 2017; Ribeiro et al., 2019], dass sich die Forschung schwerpunktmäßig auf Risikofaktoren statt auf Warnzeichen einer akuten suizidalen Krise konzentriert. Risikofaktoren und Warnzeichen unterscheiden sich hinsichtlich ihrer temporären Erstreckung: Risikofaktoren sind typischerweise langfristig mit einem erhöhten Suizidrisiko assoziiert, während Warnzeichen auf ein akutes Suizidrisiko hinweisen. Zur Verdeutlichung des Unterschieds zwischen Risikofaktoren und Warnzeichen nutzt Galynker [2017, S. 3] eine Analogie aus dem Bereich der Herzkreislauferkrankungen: Bluthochdruck, Übergewicht, Diabetes, Bewegungsmangel und ein erhöhter Cholesterinspiegel gelten als Risikofaktoren und sind prädiktiv dafür, wer im nächsten Jahrzehnt (aber nicht morgen) einen Herzinfarkt erleiden wird. Ein unmittelbar bevorstehender Herzinfarkt wird hingegen durch Warnzeichen wie Brustschmerzen, Kurzatmigkeit und Schweißbildung prädiziert. Im klinischen Alltag sind Behandler mit der Erwartung konfrontiert einzuschätzen, welcher ihrer Patienten gefährdet ist, zeitnah suizidale Handlungen durchzuführen; offensichtlich braucht es hierfür vor allem Wissen über Warnzeichen - und weniger über Risikofaktoren - einer akuten suizidalen Krise.

Von Erwin Ringel [1953] stammt eine erste Beschreibung zentraler Merkmale der psychischen Verfassung von Personen kurz vor einem Suizidversuch. Auf Basis von Interviews mit Personen, die einen Suizidversuch unternommen hatten, definierte Ringel drei Charakteristika des sogenannten präsuizidalen Syndroms: zunehmende Einengung, nach innen gerichtete Aggression und Suizidphantasien. Das präsuizidale Syndrom gilt als wichtige Beschreibung suizidaler Krisen, es mangelt jedoch an einer weitergehenden empirischen Überprüfung der Reliabilität und Validität der Syndrombeschreibung. Ein aktueller Versuch, akute Suizidalität auf Syndromebene zu definieren und empirisch abzusichern, stammt von Joiner et al. [2018]. Die Autoren postulieren ein als acute suicidal affective disturbance (ASAD) bezeichnetes Syndrom, welches durch vier Symptomgruppen definiert sein soll (Tab. 1).

Charakteristisch für ASAD ist vor allem eine drastische, zeitlich begrenzte Zuspitzung suizidaler Pläne und Absichten (increase in suicidal intent) [Tucker et al., 2016]. Aus Untersuchungen von Personen, die einen Suizidversuch überlebt haben, ist bekannt, dass die Zeiträume, innerhalb derer sich Suizidwünsche und -absichten intensivieren, kurz sind: Betroffene beschäftigen sich mit der Möglichkeit eines Suizids gegebenenfalls schon über mehrere Jahre, eine Intensivierung von Suizidgedanken findet sich dann jedoch erst im unmittelbaren Vorfeld der suizidalen Handlung [Millner et al., 2017], und insbesondere das Zeitfenster zwischen Entscheidung und Handlung ist vielfach nur von äußerst kurzer Dauer (5 Minuten
Tabelle 1. ASAD-Kriterien nach Rogers et al. [2017b]

A Drastische Zunahme der Suizidabsicht (increase of suicidal intent) innerhalb von Stunden oder Tagen (im Gegensatz zu Wochen oder Monaten)

B Eine oder beide der folgenden Erlebensweisen: soziale Entfremdung (social alienation: sozialer Rückzug, Abscheu vor anderen, Eindruck, eine Last für andere zu sein) und/ oder Selbstentfremdung (self-alienation: Selbsthass, Eindruck, sich selbst eine Last zu sein)

C Der Eindruck, dass die unter (A) und (B) beschriebenen Erlebensweisen permanent und unlösbar sind (perception of intractability)

D Zwei oder mehr Übererregungssymptome (overarousal: Agitiertheit, innere Unruhe, Schlafstörungen, Albträume, Reizbarkeit)

ASAD, acute suicidal affective disturbance.

bis wenige Stunden) [Simon et al., 2001; Rimkeviciene et al., 2015].

Unter sozialer Entfremdung (social alienation) wird vor allem ein ausgeprägter sozialer Rückzug verstanden, bisweilen gepaart mit Abscheu vor anderen Menschen. Hinzukommen kann die Überzeugung, der eigene Tod werde eine Erleichterung für das soziale Umfeld bedeuten, da man seinen Mitmenschen ohnehin nur zur Last falle. Selbstentfremdung (self-alienation) wiederum kann ihren Ausdruck in Selbsthass oder Ekel vor sich selbst finden. Das ASAD B-Kriterium bezieht offensichtlich die Kernkonstrukte der interpersonalen Theorie suizidalen Verhaltens von Joiner [2005] ein: der Eindruck, eine Last für andere zu sein (perceived burdensomeness) und der Eindruck, nicht Teil einer wertgeschätzten Gemeinschaft $\mathrm{zu}$ sein (thwarted belongingness). In einer Vielzahl von Studien ließ sich zeigen, dass beide Konstrukte von zentraler Bedeutung für das Aufkommen suizidaler Wünsche sind [Chu et al., 2017].

Hoffnungslosigkeit soll dann aufkommen, wenn Betroffenen die psychischen Belastungen als permanent und unlösbar (perception of intractability) erscheinen. Erneut wird hier auf die interpersonale Theorie suizidalen Verhaltens rekurriert, die insbesondere in ihrer revidierten Form [Van Orden et al., 2010] betont, dass das Vorhandensein von perceived burdensomeness und thwarted belongingness allein keine aktiven Suizidgedanken verursacht, sondern erst die Wahrnehmung, dass diese beiden Zustände von permanenter Natur sein werden. Während die zentrale Bedeutung von Hoffnungslosigkeit für suizidales Erleben und Verhalten in einer Vielzahl von Studien gezeigt werden konnte [McMillan et al., 2007], gibt es bislang nur wenige Untersuchungen zur Bedeutung dieser sehr spezifischen Form von Hoffnungslosigkeit [Tucker et al., 2018]. 
Übererregungssymptome (overarousal) können auf verschiedene Weise zum Ausdruck kommen: Es können Schlafstörungen wie Einschlaf- und Durchschlafschwierigkeiten oder Albträume auftreten, genauso wie innere Unruhe, Agitiertheit und/oder Reizbarkeit [Tucker et al., 2016]. Zur Bedeutung von Übererregungssymptomen für suizidales Verhalten liegen eine Vielzahl an Befunden einschließlich Erkenntnissen zu innerer Unruhe [Busch et al., 2003; Ribeiro et al., 2011], Schlafstörungen [Pigeon et al., 2012; Bernert und Nadorff, 2015], Reizbarkeit [Trivedi et al., 2011] sowie schweren affektiven Zuständen [Hendin et al., 2007, 2010] vor. Die besondere Bedeutung von Übererregungssymptomen im Vorfeld suizidaler Symptome ist vor diesem Hintergrund weitgehend unbestritten [Galynker, 2017].

Die Arbeitsgruppe um Joiner [Rogers et al., 2019a] geht davon aus, dass es sich bei ASAD um ein unidimensionales Konstrukt handelt. Die Herausforderung besteht folglich darin, ASAD als eine gültige diagnostische Entität in Form eines einheitlichen Syndroms zu bestätigen. Nach dem klassischen Ansatz von Robins und Guze [1970] zur Validierung psychiatrischer diagnostischer Entitäten sind hierzu mehrere Schritte erforderlich: (1) klinische Beschreibung, (2) Laborstudien, (3) Abgrenzung von anderen Störungsbildern, (4) Untersuchungen zum Krankheitsverlaufs und (5) familienorientierte Studien. In der klinischen Beschreibung wird das symptomale Krankheitsbild der jeweiligen Störung betrachtet. Eingeschlossen werden dabei alle Merkmale, welche die Störung, deren Prävalenz, Verlauf sowie auslösende Faktoren beeinflussen. Laborstudien umfassen physiologische, anatomische sowie psychologische Untersuchungen, die zusammengenommen den einheitlichen Störungscharakter bestätigen sollen. Die Abgrenzung von anderen Störungsbildern umfasst die Festlegung von Ausschlusskriterien, anhand derer die Unterscheidung der Störung von anderen bereits bestehenden Störungsbildern vorgenommen werden kann. Die ASAD sollte beispielsweise differenzierbar von einer Major Depression sein [Rogers et al., 2019a], und die Symptome dürfen nicht auf die Wirkung einer Substanz zurückzuführen sein [Tucker et al., 2016]. Follow-up-Studien untersuchen im Längsschnitt den Verlauf des postulierten Störungsbildes. Mit Blick auf ASAD interessiert natürlich insbesondere, ob das postulierte Störungsbild prädiktive Validität aufweist, d.h. eine Vorhersage suizidalen Verhaltens ermöglicht. In Familienstudien wird schließlich bewertet, ob eine Störung bei nahen Verwandten gehäuft auftritt - hierdurch ließen sich erste Hinweise auf eine erbliche Komponente ableiten. Eine durch diesen Prozess validierte diagnostische Entität, die akute Risikofaktoren für suizidales Verhalten in einem Syndrom abbildet, würde Klinikern die Abschätzung des akuten Suizidrisikos fundamental erleichtern und ist somit von zentraler klinischer Bedeutsamkeit.
Zur Erfassung von ASAD wurde von der Arbeitsgruppe um Joiner das Acute Suicidal Affective Disturbance Inventory-Lifetime (ASADI-L) entwickelt [Tucker et al., 2016]. Das Selbstauskunftsinstrument umfasst 28 Items zu den verschiedenen ASAD-Symptomen: Die Items erfragen eine drastische Zunahme von Suizidgedanken, -absichten und -plänen sowie mögliche Vorbereitungen auf einen Suizid. Die nachfolgenden Fragen beziehen sich auf die schwerste Episode, in der Suizidabsichten oder -pläne massiv zugenommen haben. Das Auftreten und die Schwere von Entfremdungserleben gegenüber sich selbst und anderen werden bewertet. Abschließend werden das Auftreten und die Schwere von Schlaflosigkeit, Albträumen, Agitiertheit und Reizbarkeit erfragt. Der Schweregrad von ASAD-Symptomen wird durch die Berechnung eines Summenscores abgebildet; hierzu werden 13 der 28 Items herangezogen. Die weiteren Items dienen dazu, das grundsätzliche Auftreten eines ASAD-Symptoms zu erfragen.

Vor dem Hintergrund der enormen Bedeutung der Beschreibung eines akut-suizidalen Syndroms besteht das Ziel des vorliegenden Artikels darin, die Literatur zum ASAD-Syndrom zusammenfassend darzustellen und (1) eine Einschätzung vorzunehmen, inwieweit tatsächlich von einem einheitlichen Syndrom ausgegangen werden kann, und (2) klinische Implikationen herauszuarbeiten und zu bewerten.

\section{Methodik}

Unter Verwendung der Datenbanken PubMed, PsycINFO, MEDLINE, EMBASE und Google Scholar wurde eine Literaturrecherche mit den Suchbegriffen "acute suicidal affective disturbance", "ASAD" und "akut suizidal-affektive Störung" durchgeführt. Des Weiteren wurden die Literaturverzeichnisse einschlägiger Studien nach relevanten Studien durchgesehen. Eine Beschränkung der Publikationen auf eine bestimmte Zeitspanne erfolgte nicht. Die Recherche schließt alle Studien ein, die bis April 2020 publiziert wurden.

\section{Ergebnisse}

Online-Supplement-Tabelle S1 (siehe www. karger.com/doi/10.1159/000511922 für alles OnlineSupplementmaterial) gibt einen Überblick über Studien zur akut suizidal-affektiven Störung. Insgesamt entsprachen 12 Artikel aus dem Zeitraum von 2016 bis 2020 den Suchkriterien; bei 3 dieser 12 Artikel handelte es sich um Übersichtsarbeiten [Rogers et al., 2017c; Joiner et al., 2018; Rogers et al., 2019a], die eine Diskussion der bisherigen Forschungslage darstellten und daher im Weiteren nicht berücksichtigt wurden. Final einbezogen wurden neun Artikel, von denen einer [Rogers et al., 2017b] zwei 
Studien beschreibt. Acht der neun Artikel stammen von der Arbeitsgruppe um Thomas Joiner; die meisten wurden dabei federführend von Megan Rogers publiziert. Alle Untersuchungen wurden in den USA durchgeführt; fünf Studien untersuchten Studierende und fünf Studien klinische Stichproben (ambulante und stationäre psychiatrische Patienten). Die Stichprobengrößen reichen von 58 bis 7'698 untersuchten Personen. Im Sinne der Differenzierung von Robins und Guze [1970] liegen sechs Studien zur Bestimmung der einheitlichen Störungsentität sowie vier Untersuchungen zum Ausschluss anderer Störungsbilder vor. Im Folgenden wird die Studienlage zusammenfassend dargestellt und anschließend diskutiert.

\section{Einheitliche Störungsentität und ASAD-Korrelate}

Insgesamt wurde in vier querschnittlich angelegten Studien untersucht, inwieweit es sich bei ASAD um eine einheitliche Störungsentität handelt [Stanley et al., 2016; Tucker et al., 2016; Rogers et al., 2017b, Studien 1/2], und in fünf weiteren Studien [Rogers et al., 2017a; Rogers und Joiner, 2018; Rogers und Joiner, 2019; Rogers et al., 2019c; Buckner et al., 2020] wurden ASAD-Korrelate untersucht. Zur Bestätigung wurden explorative und konfirmatorische Faktorenanalysen, multiple Regressionsanalysen sowie Analysen zur konvergenten und diskriminanten Validität durchgeführt. Sechs Studien [Tucker et al., 2016; Rogers et al. 2017a; Rogers und Joiner, 2018, 2019; Rogers et al., 2019c; Buckner et al., 2020] nutzten dabei das ASADI-L, zwei Studien [Stanley et al., 2016; Rogers et al., 2017b, Studie 1] nutzten Einzelitems aus verschiedenen Selbstauskunftsinstrumenten, und eine Studie [Rogers et al., 2017b, Studie 2] nutzte das Suicide Status Form-Interview [Jobes, 2016] zur Erfassung von ASAD. Die Studien, in welchen das ASADI-L eingesetzt wurde, zeigten durchweg eine sehr gute innere Konsistenz für dieses Selbstauskunftsinstrument (Cronbachs $\alpha=0.87-0.99$ ). Die postulierte unidimensionale Faktorenstruktur der ASAD konnte durch explorative [Tucker et al., 2016] und konfirmatorische Faktorenanalysen weitgehend bestätigt werden [Stanley et al., 2016; Rogers et al., 2017b, Studie 2]. Lediglich in einer Studie an ambulanten Patienten [Rogers et al., 2017b, Studie 1] fand sich keine Bestätigung der eindimensionalen Struktur; vielmehr wiesen die Ergebnisse auf eine zweifaktorielle Struktur hin: Faktor 1 suizidspezifische Variablen (Suizidgedanken, perceived burdensomeness, thwarted belongingness) und Faktor 2 Übererregungssymptome.

In mehreren Studien fanden sich signifikante Korrelationen zwischen ASAD-Symptomen und u.a. lebenszeitlichen Suizidversuchen, Letalität von Suizidversuchen, nichtsuizidaler Selbstverletzung, Suizidgedanken, thwarted belongingness, perceived burdensomeness, Hoffnungslosigkeit, Depressivität, Ängstlichkeit, Schlafstörungen, Agitiertheit [Tucker et al., 2016; Rogers und Joiner, 2019] sowie mit dysfunktionalen Kognitionen, Demoralisierung, negativer Emotionalität und verringerter positiver Emotionalität [Rogers et al., 2017a]. Des Weiteren zeigte sich, dass ASAD den Zusammenhang zwischen einem negativen kognitiven Stil [Rogers et al., 2019c] bzw. suizidspezifischer Rumination [Rogers und Joiner, 2018] und (lebenszeitlichen) Suizidversuchen mediiert. Buckner et al. [2020] konnten ergänzend zeigen, dass der Zusammenhang zwischen Depressivität und sozialer Ängstlichkeit auf der einen Seite und suizidalem Erleben und Verhalten auf der anderen Seite durch ASAD mediiert wird; kein entsprechender Mediationseffekt zeigte sich für Paniksymptome und schädlichen Alkoholkonsum. Schließlich konnten Rogers und Joiner [2019] zeigen, dass der Zusammenhang zwischen ASAD und (lebenszeitlichen) Suizidversuchen durch die persönliche Schmerztoleranz - erfasst mithilfe eines Druckalgometers - moderiert wird.

Grundsätzlich zeigte sich in verschiedenen Studien [Stanley et al., 2016; Tucker et al., 2016; Rogers et al., 2017b, Studien 1/2], dass das Vorliegen von ASAD differenziert zwischen Personen, die im Laufe ihres Lebens einen Suizidversuch unternommen haben, und solchen, die ausschließlich unter Suizidgedanken gelitten haben bzw. Personen, die einen versus mehrere Suizidversuche in ihrem Leben unternommen haben.

\section{Abgrenzung von anderen Störungsbildern}

Insgesamt wurde in drei Querschnittstudien [Rogers et al., 2017b, Studie 1/2; Rogers et al., 2019a] untersucht, inwieweit ASAD sich von anderen Störungsbildern [Rogers et al., 2017b] bzw. Störungssymptomatiken [Rogers et al., 2019b] differenzieren lässt. Rogers et al. [2019b] nutzten hierzu eine ASADI-Adaption zur Erfassung aktueller Symptome.

Rogers et al. [2017b, Studie 1] konnten in einer logistischen Regressionsanalyse zeigen, dass die ASAD-Diagnose prädiktiv für das Vorliegen lebenszeitlicher Suizidversuche war, während sich für keines der anderen einbezogenen Störungsbilder (u.a. Depression, posttraumatische Belastungsstörung, Zwangsstörung, Borderline-Persönlichkeitsstörung) - mit Ausnahme alkoholbezogener Störungen [vgl. Buckner et al., 2020] - ein entsprechender Zusammenhang zeigte. In einer weiteren Analyse konnten Rogers et al. [2017b, Studie 2] diesen Befund weitgehend replizieren. Mithilfe einer Netzwerkanalyse konnten Rogers et al. [2019b] schließlich zeigen, dass die ASAD-Symptome gegenüber Depressions- und Angstsymptomen ein distinktes Cluster bildeten. Die ASAD-Symptome wiesen untereinander starke Assoziationen auf, während sie nur schwache Assoziationen mit Angst- und Depressionssymptomen aufwiesen. Die höchste Zentralität innerhalb des ASAD-Clusters wiesen die Symptome sozialer Rückzug, Selbsthass und Schlafstörungen auf; hierbei scheint es sich um Kernsymptome von ASAD zu handeln. 


\section{Diskussion}

Die bisherige Befundlage zur akut suizidal-affektiven Störung stützt den postulierten Syndromcharakter: Im Rahmen mehrerer Studien ließ sich unter Verwendung unterschiedlicher Stichproben (Studierende, ambulante und stationäre Patienten), Messinstrumente (ASADI-L; Einzelitems) und statistischer Auswertungsverfahren (Faktorenanalyse, Netzwerkanalyse) zeigen, dass die vier Merkmalsgruppen (Suizidgedanken, Entfremdung, Hoffnungslosigkeit, Übererregung) im Sinne eines einheitlichen Syndroms gemeinsam auftreten und sich von anderen Störungsbildern (Depression, Angst) sicher abgrenzen lassen. Offensichtlich ist es somit gelungen, einen Zustand zu beschreiben, der suizidales Erleben in besonderem Maße charakterisiert. Hierfür spricht auch, dass die ASAD-Symptomatik zu differenzieren vermag zwischen Personen mit/ohne Suizidgedanken und solchen, die einmal oder mehrmals versucht haben, sich das Leben zu nehmen [vgl. Klonsky und May, 2014]. Darüber hinaus konnte gezeigt werden, dass die ASAD-Symptomatik sowohl prädiktiv ist für lebenszeitliche Suizidversuche - und dies unter Kontrolle diverser anderer Risikofaktoren - als auch den Zusammenhang zwischen verschiedensten Risikofaktoren und Suizidversuchen vermittelt.

Im Sinne einer guten klinische Deskription lässt sich überdies interpretieren, dass eine unabhängige Arbeitsgruppe einen sehr vergleichbaren Vorschlag zur Charakterisierung eines akut-suizidalen Zustands vorgenommen hat: Galynker [2017] beschreibt ein suicide crisis syndrome (SCS), welches durch den persistierenden Eindruck, in einer ausweglosen Situation gefangen zu sein (entrapment), affektive Symptome (emotionaler Schmerz, extreme Stimmungsschwankungen, Panik, Dissoziation), kognitive Symptome (ruminative flooding) und Übererregungssymptome (Agitiertheit, Schlafstörungen) definiert ist. Beide Syndrome beschreiben somit akute, rasch zunehmende Symptome, die suizidalem Verhalten vorausgehen sollen. Darüber hinaus ist das Vorhandensein spezifischer Symptombereiche, wie Übererregung, Hoffnungslosigkeit und sozialer Rückzug, bei beiden Syndrombeschreibungen vergleichbar. Es gibt jedoch auch Unterschiede: ASAD wird durch eine drastische Zunahme bewusst erlebter Suizidabsichten charakterisiert, während zur Diagnose des SCS keine bewusste suizidale Absicht eruierbar sein muss [Rogers et al., 2017c]. In Bezug auf die Nomenklatur erscheint die Bezeichnung eines akuten suizidalen Zustandes als suizidalaffektive Störung insofern zutreffender, da der Krisenbegriff weithin als kritischer Wendepunkt eines bereits bestehenden Krankheitsverlaufs verstanden werden kann. Der suizidal-affektive Störungsbegriff hingegen betont das Konzept einer autonomen Diagnoseentität, die maß- geblich durch affektive Merkmale (Entfremdung, Hoffnungslosigkeit, Übererregung) gekennzeichnet ist und raptusartig auftritt.

In zukünftigen Studien muss geklärt werden, welches Syndrommuster eine hilfreichere Beschreibung akut-suizidalen Erlebens ermöglicht; bislang fehlt es an vergleichenden Untersuchungen. Dies gilt gleichermaßen für die Messinstrumente, die zur Erfassung der beiden verschiedenen Syndrome genutzt werden. Das ASADI-L ist ein Selbstauskunftsinstrument, welches eine sehr gute interne Konsistenz (Cronbachs $\alpha=0.87-0.99$ ) aufweist und für das - in den meisten Studien - eine einfaktorielle Struktur nachgewiesen wurde. Die Suicide Trigger Scale (STS) [Yaseen et al., 2010], welche zur Erfassung des SCS genutzt wird, nutzt ebenfalls die Selbstbeurteilung des Patienten, untergliedert sich jedoch in drei Subskalen: frantic hopelessness (rasende Hoffnungslosigkeit), ruminative flooding (ruminative Überflutung) und near psychotic somatization (psychosenahe Somatisierung). Die aktuelle dritte Überarbeitung dieses Instruments (STS-3) [Yaseen et al., 2014] zeigte in einer Studie zum prospektiven Potential eine Überlegenheit gegenüber anderen Instrumenten zur Erfassung von Psychopathologie (z.B. BSI) oder Suizidalität (z.B. C-SSRS), konnte jedoch aktuelle Suizidversuche, die im Vorfeld der Befragung verübt worden waren, nicht vorhersagen. Vergleichende Untersuchungen der beiden Messinstrumente - u.a. zur Erfassung der konvergenten Validität - stehen bislang aus.

Grundsätzlich wäre ein genaues Wissen um einen $\mathrm{Zu}$ stand akuter Suizidalität in der klinischen Praxis von unschätzbarem Wert und würde die Risikoabschätzung suizidaler Patienten erheblich erleichtern: Im Rahmen der Risikoabschätzung müsste vordringlich bestimmt werden, ob sich die akut suizidal-affektive Störung diagnostizieren lässt. Lässt sich die Störung diagnostizieren, so ist von einer akuten Gefährdung der Person auszugehen und es liegt nahe, Betroffene in ein geschütztes Setting zu überführen. Zum jetzigen Zeitpunkt ist die ASAD-Diagnose aber mitnichten so abgesichert, dass sie ein solches Vorgehen rechtfertigen würde. Der aktuelle Forschungsstand erlaubt die Feststellung eines einheitlichen Störungsbildes; bislang liegen aber keinerlei Befunde dazu vor, ob dieses Störungsbild tatsächlich suizidalem Verhalten vorausgeht. Im Sinne des Forschungsschemas zur Validierung von Diagnosen sensu Robins und Guze [1970] fehlt es somit an Studien zum Krankheitsverlauf. In ersten Studien könnten dazu Interviews mit Personen nach einem Suizidversuch durchgeführt werden oder psychologische Autopsiestudien, bei denen Behandler und Angehörige zum Vorliegen von ASAD im unmittelbaren Vorfeld der suizidalen Handlung befragt werden [vgl. Hawton et al., 1998]. In weiteren Studien muss sodann die prädiktive Bedeutung des Vorliegens einer ASAD-Diagnose für das prospektive Auftreten suizidaler 
Handlungen untersucht werden. Erst dann, wenn sich das Syndrom sicher als "Warnzeichen" suizidalen Verhaltens erwiesen hat, wird es von klinischem Nutzen sein.

Aus verschiedenen Gründen lässt sich jedoch anzweifeln, dass dies gelingen kann: Zwar ließ sich für sämtliche ASAD-Einzelsymptome zeigen, dass diese prädiktiv für suizidales Verhalten sind [Bernert und Joiner, 2007; Ribeiro et al., 2016; Rogers et al., 2016; Chu et al., 2017], die gefundenen Zusammenhänge sind aber bestenfalls von moderater Größe. In diesem Sinne zeigte sich in der Metaanalyse von Franklin et al. [2017], dass es - auf der Basis von Einzelprädiktoren (wie z.B. dem Vorliegen von Suizidgedanken) - nicht möglich ist, suizidales Verhalten besser als auf Zufallsniveau vorherzusagen. Und auch Faktoren wie der Eindruck, eine Last für andere zu sein, oder Agitiertheit weisen nur einen moderaten Zusammenhang mit späterem suizidalen Verhalten auf [Rogers et al., 2016; Chu et al., 2017]. Allerdings ist nicht auszuschließen, dass der ASAD-Symptomkombination größere prädiktive Bedeutung zukommen mag als den ASADEinzelsymptomen. In einer Prädiktionsanalyse, die kürzlich zum SCS publiziert wurde [Yaseen et al., 2019], ließ sich beispielsweise zeigen, dass das Vorliegen des SCS zwar der stärkste Prädiktor für das Auftreten eines Suizidversuchs in den ersten 4-8 Wochen nach einem stationären Aufenthalt war; die Klassifikationsgenauigkeit war gleichwohl gering. So wurde gerade einmal bei der Hälfte derer, die einen Suizidversuch unternahmen, vorab das SCS diagnostiziert.

Skeptisch stimmt zudem eine aktuelle Studie von Huang et al. [2020], in welcher - mithilfe maschinellen Lernens - gezeigt werden konnte, dass für die Differenzierung von Personen mit oder ohne lebenszeitlichen Suizidversuch keinem einzelnen Konstrukt besondere Bedeutung zukommt; vielmehr konnten die unterschiedlichsten Symptomkombinationen zur Differenzierung der beiden Personengruppen herangezogen werden. Es mag also sein, dass suizidales Verhalten auf sehr vielen unterschiedlichen Wegen zustande kommt und eine Reduktion auf einzelne Symptommuster immer zu kurz greifen wird.

Letztlich handelt es sich hierbei aber um eine empirische Frage. Die bisherigen Studien zu ASAD legen nahe, dass sich weitere Untersuchung zur prädiktiven Bedeutung des Syndroms lohnen. Und natürlich wäre bereits eine kleine Verbesserung der Vorhersagegenauigkeit suizidalen Verhaltens von großem klinischen Wert [vgl. Whiting und Fazel, 2019]. Gewarnt sei ausschließlich vor einer vorschnellen und ausschließlichen Ausrichtung der Risikoabschätzung auf Merkmale der akut suizidal-affektiven Störung.

\section{Statement of Ethics}

Da es sich um eine reine Übersichtsarbeit zu bestehender Literatur handelt, gab es keine ethischen Aspekte, für die ein Ethikantrag hätte gestellt werden müssen.

\section{Conflict of Interest Statement}

Die Autoren erklären, dass kein Interessenskonflikt besteht, der die hier dargestellten Inhalte beeinflusst haben könnte.

\section{Funding Sources} führt.

Die Untersuchung wurde ohne finanzielle Förderung durchge-

\section{Author Contributions}

C. Claus hat die erste Manuskriptfassung geschrieben und die Datenerhebung durchgeführt. T. Teismann hat die finale Manuskriptfassung erstellt.

\section{Literatur}

Bernal M, Haro JM, Bernert S, Brugha T, de Graaf $\mathrm{R}$, Bruffaerts R, et al.; ESEMED/MHEDEA Investigators. Risk factors for suicidality in Europe: results from the ESEMED study. J Affect Disord. 2007 Aug;101(1-3):27-34.

Bernert RA, Joiner TE. Sleep disturbances and suicide risk: A review of the literature. Neuropsychiatr Dis Treat. 2007 Dec;3(6):735-43.

Bernert RA, Nadorff MR. Sleep disturbances and suicide risk. Sleep Med Clin. 2015 Mar;10(1): 35-9.

Buckner JD, Lewis EM, Tucker RP. Mental health problems and suicide risk: the impact of acute suicidal affective disturbance. Arch Suicide Res. 2020;24(Suppl 1):303-13.
Busch KA, Fawcett J, Jacobs DG. Clinical correlates of inpatient suicide. J Clin Psychiatry. 2003 Jan;64(1):14-9.

Chu C, Buchman-Schmitt JM, Stanley IH, Hom MA, Tucker RP, Hagan CR, et al. The interpersonal theory of suicide: A systematic review and meta-analysis of a decade of crossnational research. Psychol Bull. 2017 Dec; 143(12):1313-45.

Franklin JC, Ribeiro JD, Fox KR, Bentley KH, Kleiman EM, Huang X, et al. Risk factors for suicidal thoughts and behaviors: A metaanalysis of 50 years of research. Psychol Bull. 2017 Feb;143(2):187-232.

Galynker I. The suicidal crisis. New York: Oxford University Press; 2017.
Hawton K, Appleby L, Platt S, Foster T, Cooper J, Malmberg A, et al. The psychological autopsy approach to studying suicide: a review of methodological issues. J Affect Disord. 1998 Sep;50(2-3):269-76.

Hendin H, Al Jurdi RK, Houck PR, Hughes S, Turner JB. Role of intense affects in predicting short-term risk for suicidal behavior: a prospective study. J Nerv Ment Dis. 2010 Mar; 198(3):220-5.

Hendin H, Maltsberger JT, Szanto K. The role of intense affective states in signaling a suicide crisis. J Nerv Ment Dis. 2007 May; 195(5): 363-8. 
Huang X, Ribeiro JD, Franklin JC. The differences between suicide ideators and suicide attempters: simple, complicated, or complex? J Consult Clin Psychol. 2020 Jun;88(6):554-69.

Jobes D. Managing suicidal risk: a collaborative approach. New York: Guilford Press; 2016.

Joiner TE. Why people die by suicide. Cambridge: Harvard University Press; 2005.

Joiner TE, Simpson S, Rogers ML, Stanley IH, Galynker II. Whether Called Acute Suicidal Affective Disturbance or Suicide Crisis Syndrome, a Suicide-specific Diagnosis Would Enhance Clinical Care, Increase Patient Safety, and Mitigate Clinician Liability. J Psychiatr Pract. 2018 Jul;24(4):274-8.

Klonsky ED, May AM. Differentiating suicide attempters from suicide ideators: a critical frontier for suicidology research. Suicide Life Threat Behav. 2014 Feb;44(1):1-5.

McMillan D, Gilbody S, Beresford E, Neilly L. Can we predict suicide and non-fatal self-harm with the Beck Hopelessness Scale? A metaanalysis. Psychol Med. 2007 Jun;37(6):76978 .

Millner AJ, Lee MD, Nock MK. Describing and measuring the pathway to suicide attempts: a preliminary study. Suicide Life Threat Behav. 2017 Jun; 47(3):353-69.

Nock MK, Borges G, Ono Y. Suicide. Global perspectives from the WHO World Mental Health Survey. Cambridge: Cambridge University Press; 2012.

Pigeon WR, Pinquart M, Conner K. Meta-analysis of sleep disturbance and suicidal thoughts and behaviors. J Clin Psychiatry. 2012 Sep; 73(9):e1160-7.

Ribeiro JD, Bender TW, Selby EA, Hames JL, Joiner TE. Development and validation of a brief self-report measure of agitation: the Brief Agitation Measure. J Pers Assess. 2011 Nov;93(6):597-604.

Ribeiro JD, Franklin JC, Fox KR, Bentley KH, Kleiman EM, Chang BP, et al. Self-injurious thoughts and behaviors as risk factors for future suicide ideation, attempts, and death: a meta-analysis of longitudinal studies. Psychol Med. 2016 Jan;46(2):225-36.

Ribeiro J, Huang X, Fox KR, Walsh CG, Linthicum KP. Predicting imminent suicidal thoughts and nonfatal attempts: the role of complexity. Clin Psychol Sci. 2019;7(5):94157.

Rimkeviciene J, O’Gorman J, De Leo D. Impulsive suicide attempts: a systematic literature review of definitions, characteristics and risk factors. J Affect Disord. 2015 Jan;171:93-104.
Ringel E. Der Selbstmord. Abschluß einer krankhaften Entwicklung. Wien, Düsseldorf: Maudrich; 1953.

Robins E, Guze SB. Establishment of diagnostic validity in psychiatric illness: its application to schizophrenia. Am J Psychiatry. 1970 Jan; 126(7):983-7.

Rogers ML, Anestis JC, Harrop TM, Schneider M, Bender TW, Ringer FB, et al. Examination of MMPI-2-RF Substantive Scales as Indicators of Acute Suicidal Affective Disturbance Components. J Pers Assess. 2017a Jul-Aug;99(4): 424-34.

Rogers ML, Chiurliza B, Hagan CR, Tzoneva M, Hames JL, Michaels MS, et al. Acute suicidal affective disturbance: factorial structure and initial validation across psychiatric outpatient and inpatient samples. J Affect Disord. 2017b Mar;211:1-11.

Rogers ML, Chu C, Joiner T. The necessity, validity, and clinical utility of a new diagnostic entity: acute suicidal affective disturbance. J Clin Psychol. 2019a Jun;75(6):999-1010.

Rogers ML, Galynker II, Yaseen Z, DeFazio K, Joiner TE. An overview and comparison of two proposed suicide-specific diagnoses: acute suicidal affective disturbance and suicide crisis syndrome. Psychiatr Ann. 2017c; 8(8):416-20.

Rogers ML, Hom MA, Joiner TE. Differentiating acute suicidal affective disturbance (ASAD) from anxiety and depression Symptoms: A network analysis. J Affect Disord. 2019b May; 250:333-40.

Rogers ML, Joiner TE. Lifetime acute suicidal affective disturbance symptoms account for the link between suicide-specific rumination and lifetime past suicide attempts. J Affect Disord. 2018 Aug;235:428-33.

Rogers ML, Joiner TE. Interactive Effects of Acute Suicidal Affective Disturbance and Pain Persistence on Suicide Attempt Frequency and Lethality. Crisis. 2019 Nov;40(6):413-21.

Rogers ML, Ringer FB, Joiner TE. A meta-analytic review of the association between agitation and suicide attempts. Clin Psychol Rev. 2016 Aug;48:1-6.

Rogers ML, Tucker RP, Law KC, Bauer BW, Smith CE, Capron DW, et al. The Relationship Between Negative Cognitive Styles and Lifetime Suicide Attempts is Indirect Through Lifetime Acute Suicidal Affective Disturbance Symptoms. Cognit Ther Res. 2019c;2(2):35464
Simon OR, Swann AC, Powell KE, Potter LB, Kresnow MJ, O'Carroll PW. Characteristics of impulsive suicide attempts and attempters. Suicide Life Threat Behav. 2001;32(1 Suppl): 49-59.

Stanley IH, Rufino KA, Rogers ML, Ellis TE, Joiner TE. Acute Suicidal Affective Disturbance (ASAD): A confirmatory factor analysis with 1442 psychiatric inpatients. J Psychiatr Res. 2016 Sep;80:97-104.

Statistisches Bundesamt. Todesursachenstatistik. 2020. www.destatis.de.

Trivedi MH, Wisniewski SR, Morris DW, Fava M, Kurian BT, Gollan JK, et al. Concise Associated Symptoms Tracking scale: a brief selfreport and clinician rating of symptoms associated with suicidality. J Clin Psychiatry. 2011 Jun;72(6):765-74.

Tucker RP, Hagan CR, Hill RM, Slish ML, Bagge CL, Joiner TE Jr, et al. Empirical extension of the interpersonal theory of suicide: investigating the role of interpersonal hopelessness. Psychiatry Res. 2018 Jan;259:427-32.

Tucker RP, Michaels MS, Rogers ML, Wingate LR, Joiner TE Jr. Construct validity of a proposed new diagnostic entity: Acute Suicidal Affective Disturbance (ASAD). J Affect Disord. 2016 Jan;189:365-78.

Van Orden KA, Witte TK, Cukrowicz KC, Braithwaite SR, Selby EA, Joiner TE Jr. The interpersonal theory of suicide. Psychol Rev. 2010 Apr;117(2):575-600.

Whiting D, Fazel S. How accurate are suicide risk prediction models? Asking the right questions for clinical practice. Evid Based Ment Health. 2019 Aug;22(3):125-8.

WHO. Preventing suicide. A global imperative. Genf: WHO Press; 2014.

Yaseen ZS, Hawes M, Barzilay S, Galynker I. Predictive validity of proposed diagnostic criteria for the suicide crisis syndrome: an acute presuicidal state. Suicide Life Threat Behav. 2019 Aug;49(4):1124-35.

Yaseen Z, Katz C, Johnson MS, Eisenberg D, Cohen LJ, Galynker II. Construct development: the Suicide Trigger Scale (STS-2), a measure of a hypothesized suicide trigger state. BMC Psychiatry. 2010 Dec;10(1):110.

Yaseen ZS, Kopeykina I, Gutkovich Z, Bassirnia A, Cohen LJ, Galynker II. Predictive validity of the Suicide Trigger Scale (STS-3) for postdischarge suicide attempt in high-risk psychiatric inpatients. PLoS One. 2014 Jan; 9(1):e86768. 